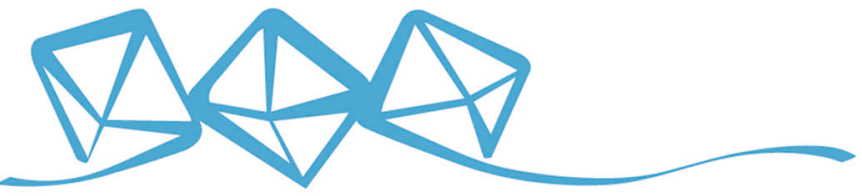 COMMUNICATIONS MATERIALS
}

ARTICLE

https://doi.org/10.1038/s43246-020-0028-z

OPEN

\section{Hysteresis-less and stable perovskite solar cells with a self-assembled monolayer}

\author{
Ganbaatar Tumen-Ulzii (1) 1,2, Toshinori Matsushima (1) 1,2,3, Dino Klotz (10 3 ${ }^{3}$, Matthew R. Leyden ${ }^{1,2}$, \\ Pangpang Wang ${ }^{4}$, Chuanjiang Qin ${ }^{5}$, Jin-Wook Lee (D) ${ }^{6}$, Sung-Joon Lee $\mathbb{D}^{6}{ }^{6}$, Yang Yang ${ }^{6}$ \& \\ Chihaya Adachi iD 1,2,3,4凶
}

Organic-inorganic halide perovskites are promising for use in solar cells because of their efficient solar power conversion. Current-voltage hysteresis and degradation under illumination are still issues that need to be solved for their future commercialization. However, why hysteresis and degradation occur in typical perovskite solar cell structures, with an electron transport layer of metal oxide such as $\mathrm{SnO}_{2}$, has not been well understood. Here we show that one reason for the hysteresis and degradation is because of the localization of positive ions caused by hydroxyl groups existing at the $\mathrm{SnO}_{2}$ surface. We deactivate these hydroxyl groups by treating the $\mathrm{SnO}_{2}$ surface with a self-assembled monolayer. With this surface treatment method, we demonstrate hysteresis-less and highly stable perovskite solar cells, with no degradation after $1000 \mathrm{~h}$ of continuous illumination.

\footnotetext{
${ }^{1}$ Center for Organic Photonics and Electronics Research (OPERA), Kyushu University, 744 Motooka, Nishi, Fukuoka 819-0395, Japan. ${ }^{2}$ Japan Science and Technology Agency (JST), ERATO, Adachi Molecular Exciton Engineering Project, 744 Motooka, Nishi, Fukuoka 819-0395, Japan. ${ }^{3}$ International Institute for Carbon-Neutral Energy Research (WPI-I2CNER), Kyushu University, 744 Motooka, Nishi, Fukuoka 819-0395, Japan. ${ }^{4}$ Institute of Systems, Information Technologies and Nanotechnologies (ISIT), Fukuoka Industry-Academia Symphonicity (FiaS), 4-1 Kyudaishinmachi, Nishi, Fukuoka 819-0388, Japan. ${ }^{5}$ State Key Laboratory of Polymer Physics and Chemistry, Changchun Institute of Applied Chemistry, Chinese Academy of Sciences, 5625 Renmin Street, 130022 Changchun, China. ${ }^{6}$ Department of Materials Science and Engineering and California NanoSystems Institute, University of California, Los Angeles, CA 90095, USA. ${ }^{凶}$ email: adachi@cstf.kyushu-u.ac.jp
} 
O rganic-inorganic lead halide perovskites have many excellent advantages, such as high absorption coefficient, bandgap tunability, long carrier diffusion length, high hole and electron mobilities, and compatibility with low-cost solution processing ${ }^{1-6}$. By taking these advantages, perovskites are used as the light absorber of solar cells. The highest certified power conversion efficiency (PCE) of perovskite solar cells (PSCs) has already reached $25.2 \%{ }^{7}$, which is comparable to silicon solar cell technology. However, because perovskites are composed of many kinds of ions, the ion migration through PSCs is a potential obstacle for efficient and stable solar power conversion in PSCs. The ion migration frequently causes current density-voltage $(J-$ $V)$ hysteresis and reduced operational stability ${ }^{8-11}$. Various approaches have been employed to reduce the ion migration. The reported approaches include compositional engineering of perovskite materials ${ }^{12}$, reducing grain boundary density by increasing grain size $\mathrm{e}^{13,14}$, and passivation of grain boundaries using organic materials ${ }^{6,13}$. However, the current situation is still far from completely blocking the ion migration in perovskite materials and devices ${ }^{11,15}$. It is also necessary to clarify mechanisms of the ion migration behind.

Weber et al. ${ }^{16}$ recently reported that the localization of positively charged ions at the interface between the perovskite layer and the electron transport layer (ETL) causes the $J-V$ hysteresis in $\mathrm{PSCs}^{16}$. The localized positive ions accelerate non-radiative recombination, leading to the quick degradation of PSCs as well ${ }^{16-18}$. Here, the positive ions are defined as all possible ions and vacancies. However, why the localization of the positive ions happens is still unclear.

Herein, we report that the presence of hydroxyl $(-\mathrm{OH})$ groups at a metal oxide ETL is one possible origin of this ionic localization. Metal oxides like $\mathrm{SnO}_{2}$ used in this study as the ETL typically have the $-\mathrm{OH}$ groups on the surfaces ${ }^{19}$. To avoid the localization of the positive ions, we deactivate the $-\mathrm{OH}$ groups by chemically modifying the $\mathrm{SnO}_{2}$ surface with a self-assembled monolayer (SAM) of a fullerene derivative. Introducing this interfacial SAM significantly reduces the $J-V$ hysteresis. With this SAM treatment, almost no degradation of PSCs is obtained at room temperature under continuous illumination for $1000 \mathrm{~h}$.

\section{Results and discussion}

Ion migration and hysteresis. To identify the existence of the ion migration, temperature-dependent electrical conductivity measurements were carried out by measuring current-voltage $(I-V)$ curves of perovskite films in a lateral-electrode structure. This measurement was designed to highlight ionic conduction, as ions would not contribute to the conductivity at low temperatures, but would contribute when the temperature was sufficiently high to provide the energy required to form mobile ions ${ }^{20}$. Figure 1a shows $I-V$ curves and revealed that the hysteresis increased with increasing temperature. This result indicates the existence of the ion migration in our perovskite films and is consistent with a previous report ${ }^{21-24}$.

We fabricated planar PSCs with a regular structure of glass substrate/indium tin oxide (ITO) $/ \mathrm{SnO}_{2} /$ perovskite $/ 2,2^{\prime}, 7,7^{\prime}$-tetrakis[N,N-di(4-methoxyphenyl)amino]-9,9'-spirobifluorene (spiro$\mathrm{OMeTAD}) / \mathrm{Au}$ and measured their $J-V$ curves. Here we defined the voltage scan from -0.1 to $1.2 \mathrm{~V}$ as the forward scan and the voltage scan from 1.2 to $-0.1 \mathrm{~V}$ as the reverse scan. In this study, we carried out the consecutive forward-to-reverse scans for the PSC evaluation, unless otherwise mentioned. Eight devices fabricated in the same batch had the average PCEs of $17.4 \%$ and $17.7 \%$ in the forward and reverse scans, respectively, at the scan rate of $200 \mathrm{mV} \mathrm{s}^{-1}$ (Supplementary Table 1). The $J-V$ hysteresis seemed very small at this scan rate, and the hysteresis index (HI) defined in the "Methods" section was close to zero. This small hysteresis is consistent with literature, in which $\mathrm{SnO}_{2}$ was used as the ETL in PSCs ${ }^{25,26}$. However, the hysteresis increased with decreasing the scan rates from 200 to $10 \mathrm{mV} \mathrm{s}^{-1}$ (Fig. $1 \mathrm{~b}$ and Supplementary Table 2). The negative HI values obtained at the lower scan rates mean "inverted hysteresis," for which the PCE is higher in the forward scan than in the reverse scan.

It has been reported that one of the relevant origins of the inverted hysteresis is the ion migration in PSCs $9,27,28$. The migration of different ionic species in perovskite films could cause the $J-V$ hysteresis, the degree of which depends on the voltage scan rates ${ }^{9}$, directions (forward-to-reverse or reverse-to-forward bias scans $)^{29}$, ranges ${ }^{27,30}$, and temperature ${ }^{31}$. Thus, we measured $J-V$ curves in the consecutive reverse-to-forward scans (Supplementary Fig. 1a), which are opposite to the forward-to-reverse scans we used earlier (Supplementary Fig. 1b). The scan rate was $10 \mathrm{mV} \mathrm{s}^{-1}$. In this case, the reverse scan is better than the forward scan in terms of the PCE. Eventually, the scan directions are not so important; the first scan provides the higher PCE and repeating the scans decreased the PCEs. Next, we measured $J-V$ curves in a wider scan range between -0.1 and $2.0 \mathrm{~V}$ at a scan rate of $200 \mathrm{mV} \mathrm{s}^{-1}$ (Supplementary Fig. 2). The hysteresis began to appear with this scan range, although the hysteresis was not clearly observed when we used the small scan range from -0.1 to $1.2 \mathrm{~V}$ (Fig. 1b). This may mean that applying a high voltage to PSCs caused more serious hysteresis. For a better understanding of the temperature effect on the hysteresis, we measured $J-V$ curves at a high temperature of $60^{\circ} \mathrm{C}$ at a scan rate of $200 \mathrm{mV} \mathrm{s}^{-1}$, resulting in the larger $J-V$ hysteresis (Fig. 1c). The hysteresis again decreased after cooling the PSCs to room temperature (Fig. 1d). Considering the aforementioned experimental results, the ion migration is likely in our PSCs.

Detection of $-\mathrm{OH}$ groups on a $\mathrm{SnO}_{2}$ surface. We speculate that the $-\mathrm{OH}$ groups attract the positive ions existing near the $\mathrm{SnO}_{2}$ surface in our PSCs, although most of the positive ions move to the opposite spiro-OMeTAD side along the internal built-in field. The $-\mathrm{OH}$ groups were previously identified from the $\mathrm{SnO}_{2}$ surface 32,33 . To observe the $-\mathrm{OH}$ groups, we characterized the $\mathrm{SnO}_{2}$ surface with X-ray photoelectron spectroscopy (XPS). The $\mathrm{Sn} 3 d$ core level peak was detected (Fig. 2a). By analyzing the $\mathrm{O} 1 s$ core level region (Fig. 2b), an XPS peak originating from the $-\mathrm{OH}$ groups was identified at $531.7 \mathrm{eV}$ near a main oxygen peak at $530.2 \mathrm{eV}$. This is proof of the presence of the $-\mathrm{OH}$ groups on the $\mathrm{SnO}_{2}$ surface ${ }^{19,34,35}$, which is expected to induce the localization of the positive ions.

Electrochemical impedance spectroscopy (EIS) measurements were carried out on the PSCs to characterize the interfacial charge dynamics caused by the localized positive ions. Figure $2 \mathrm{c}$ shows EIS data recorded at an open-circuit voltage $\left(V_{\mathrm{OC}}\right)$ condition under illumination. The EIS measurements consisted of a series of three consecutive measurements to check the consistency of the results during the entire EIS measurement procedure. The highfrequency semicircle for every consecutive spectrum increased. The low-frequency impedance did not have a clear pattern and did not change in shape or magnitude throughout the EIS measurement procedure. The increase in impedance at $0.07 \mathrm{~Hz}$ (lowest measured frequency) from 12.2 to $13.8 \Omega \mathrm{cm}^{2}$ was predominantly caused by the high-frequency semicircle. This feature was previously attributed to poor electron extraction or recombination of charge carriers at the interfaces between the charge transport layer and the perovskite layer ${ }^{36}$. Recently, we reported that the short-term increase in high-frequency impedance is $\sim 100 \%$ reversible by keeping the PSC in the dark ${ }^{37}$. This 

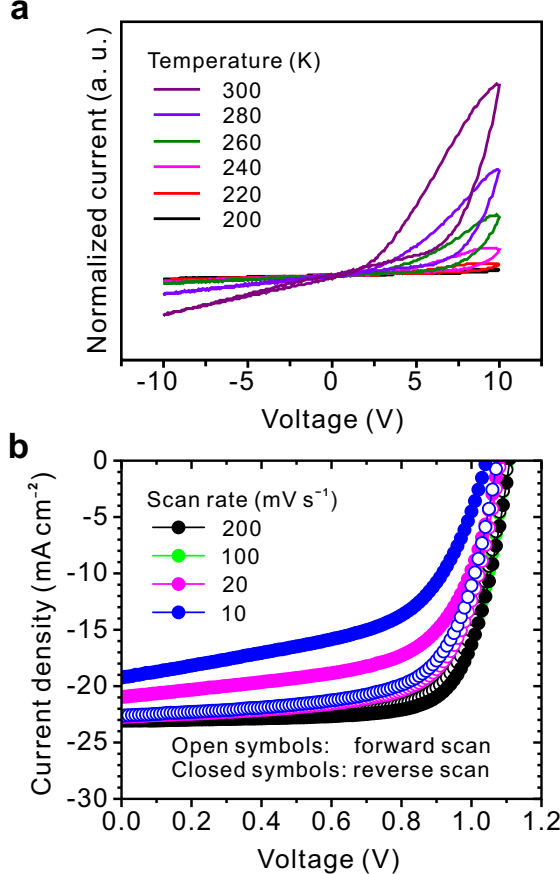
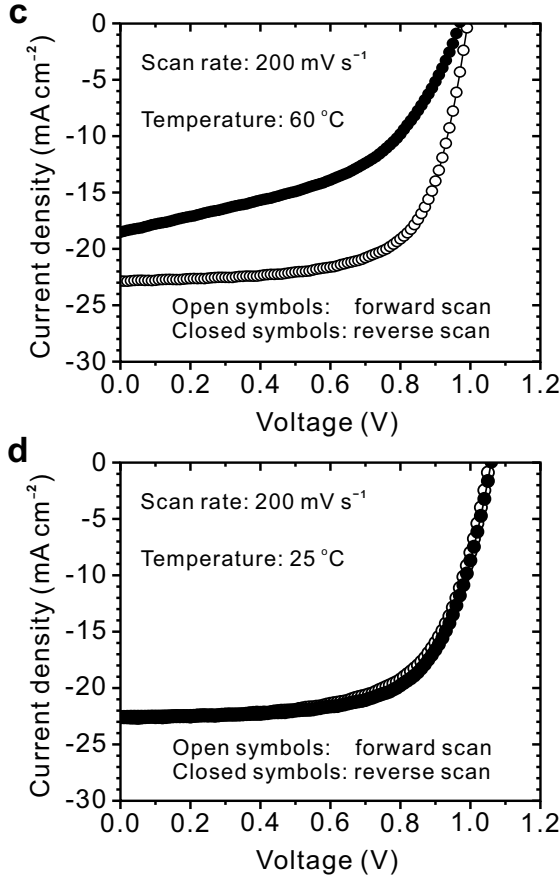

Fig. 1 Indication of ion migration in perovskite films and perovskite solar cells. a Temperature-dependent conductivity measurements in a lateral structured perovskite film. b Scan-rate-dependent current density-voltage curves of perovskite solar cells. $\mathbf{c}$, $\mathbf{d}$ Effect of temperature on the hysteresis of perovskite solar cells at a fast scan rate of $200 \mathrm{mV} \mathrm{s}^{-1}$. Current density-voltage curves measured at $60^{\circ} \mathrm{C}$ (c) and after cooling to room temperature (d).
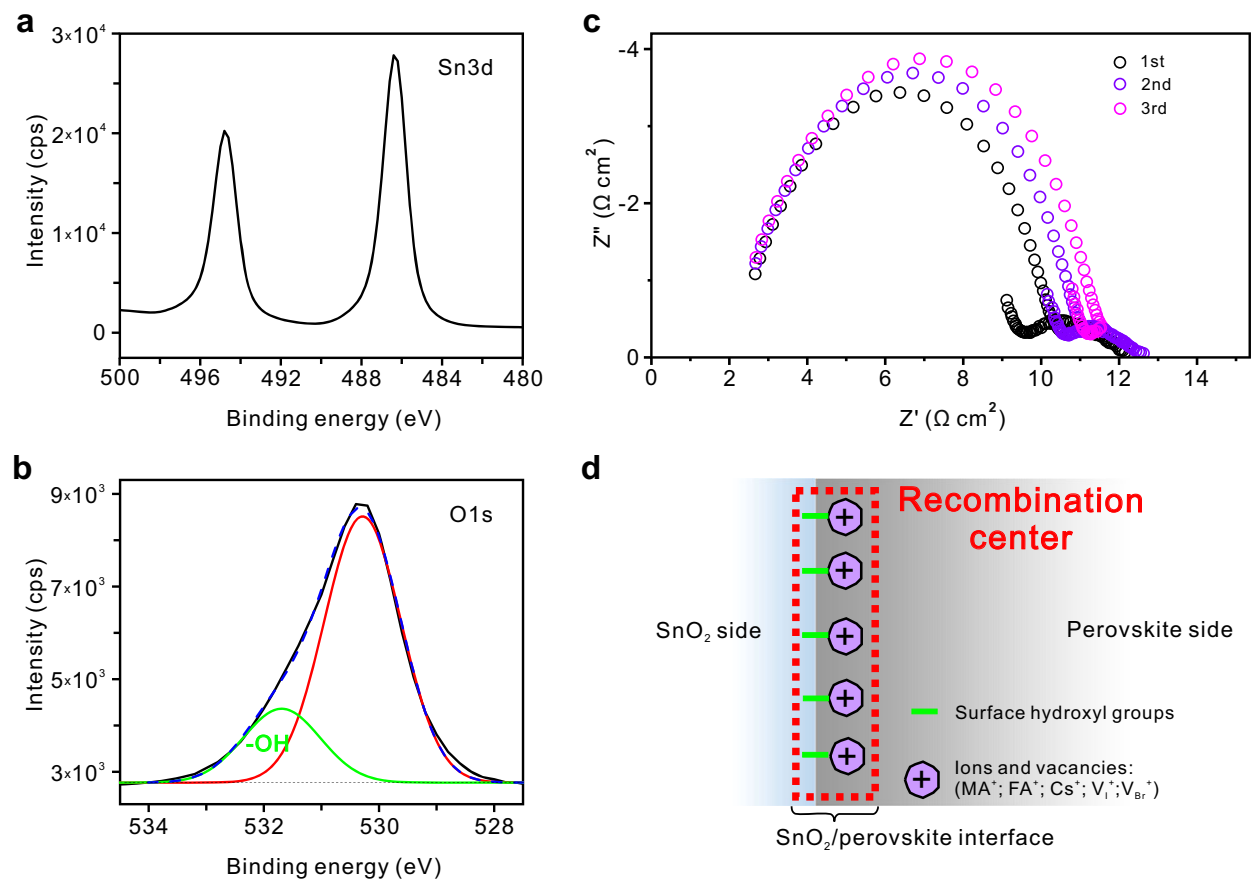

d

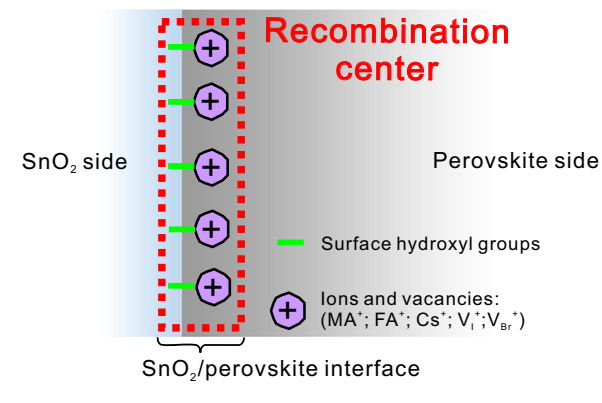

Fig. 2 Presence of surface charges on a $\mathrm{SnO}_{2}$ surface induces ion accumulation and, therefore, increases carrier recombination in perovskite solar cells. a, b X-ray photoelectron spectroscopy peaks of a $\mathrm{SnO}_{2}$ thin film in the $\mathrm{Sn} 3 d$ (a) and $\mathrm{O}$ 1s (b) regions. c Electrochemical impedance spectroscopy results of perovskite solar cells. The electrochemical impedance spectroscopy measurements were repeated three times. $\mathbf{d}$ Illustration showing the positive ion localization caused by the surface-negative charges $(-\mathrm{OH})$ of $\mathrm{SnO}_{2}$. The possible mobile positive ions are $\mathrm{MA}^{+}$(methylammonium ion), $\mathrm{FA}^{+}$ (formamidinium ion), $\mathrm{V}_{1}+$ (iodine vacancy), and $\mathrm{V}_{\mathrm{Br}}{ }^{+}$(bromine vacancy).

feature was related to the ion migration and accumulation, which leads to the increased non-radiative recombination ${ }^{37}$. Therefore, we attributed the short-term increase in high-frequency impedance in Fig. $2 \mathrm{c}$ to the localized ionic charges at the negatively charged surface of $\mathrm{SnO}_{2}$. The localized and accumulated ions, including charged vacancies (especially, iodine vacancies), are well known to act as trap-assisted charge recombination centers in PSCs ${ }^{17,28,38,39}$. Therefore, electron extraction to $\mathrm{SnO}_{2}$ was reduced because of the trap-assisted recombination, as depicted in Fig. 2d, resulting in the large hysteresis. 
a

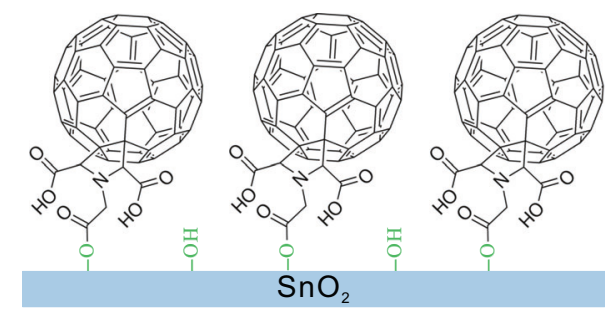

C

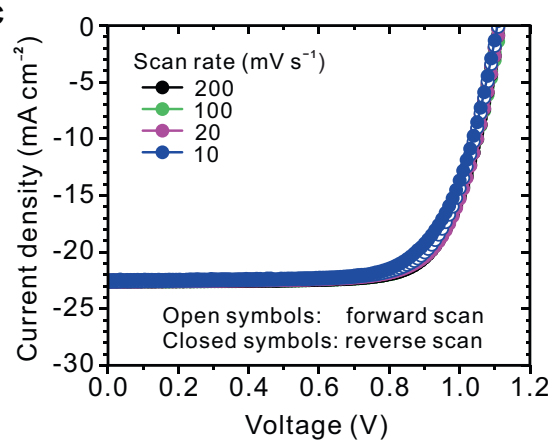

e

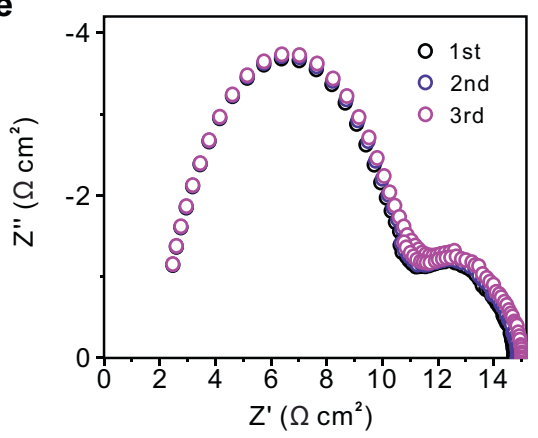

b

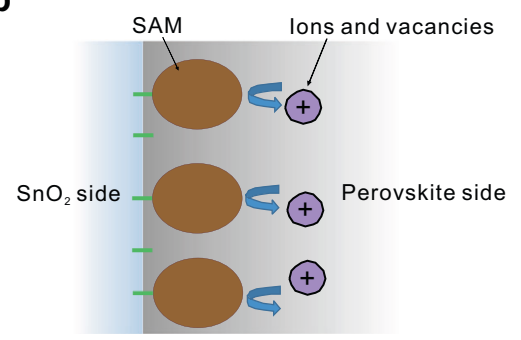

d

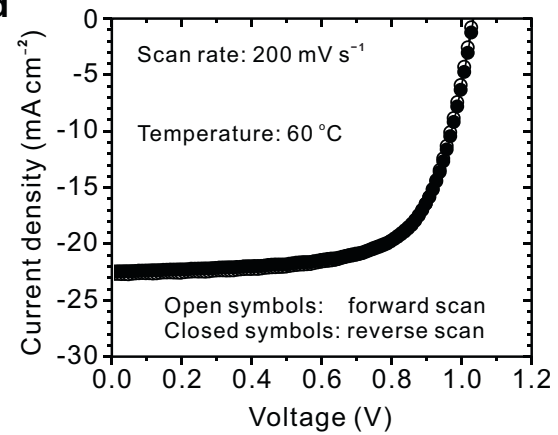

f

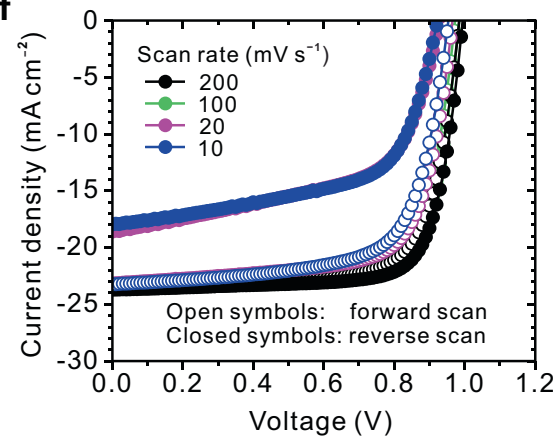

Fig. 3 Positive effect of using a self-assembled monolayer (SAM) on the hysteresis and carrier recombination characteristics in perovskite solar cells. Illustration showing a a self-assembled monolayer formed on the $\mathrm{SnO}_{2}$ surface and $\mathbf{b}$ reduced localized ionic charges at the surface of $\mathrm{SnO}_{2}$ because of the deactivation of surface - $\mathrm{OH}$ groups. c Scan-rate-dependent current density-voltage curves of perovskite solar cells with a self-assembled monolayer at room temperature. d Current density-voltage curves measured at a fast scan rate of $200 \mathrm{mV} \mathrm{s}^{-1}$ at $60^{\circ} \mathrm{C}$. e Electrochemical impedance spectroscopy results of perovskite solar cells with a self-assembled monolayer. $\mathbf{f}$ Scan-rate-dependent current density-voltage curves of perovskite solar cells with a $\mathrm{C}_{60}$ layer at room temperature.

Surface treatment with a SAM. To deactivate the surface $-\mathrm{OH}$ groups, the $\mathrm{SnO}_{2}$ layer was modified by a SAM of a fullerene $\left(\mathrm{C}_{60}\right)$ derivative (Fig. 3a). The $\mathrm{C}_{60}$ derivative used here was $\mathrm{C}_{60}$ pyrrolidine tris-acid (CPTA) because this material has the ability to form strong chemical bonding with the $\mathrm{SnO}_{2}$ surface ${ }^{40,41}$ and to behave as a good electron acceptor. The CPTA-SAM was formed on the $\mathrm{SnO}_{2}$ surface by spin coating from $N, N$-dimethylformamide (DMF) solution. The existence of the CPTA-SAM was confirmed by the appearance of XPS peaks from the $\mathrm{C} 1 \mathrm{~s}$ and $\mathrm{N} 1 s$ core levels and the decreased intensities of XPS peaks from the Sn $3 d$ and O $1 s$ core levels (Supplementary Fig. 3). Even after washing the substrates with DMF, the intensities of all the XPS peaks from the $\mathrm{C} 1 s, \mathrm{~N} 1 s, \mathrm{Sn} 3 d$, and $\mathrm{O} 1 s$ core levels were unchanged (Supplementary Fig. 3), indicating that most of the CPTA molecules are chemically bounded on the $\mathrm{SnO}_{2}$ surface. With the CPTA-SAM formation, the -COO- groups were formed through the esterification reaction ${ }^{40,42}$ between the CPTA molecules and the surface - $\mathrm{OH}$ groups. This was supported by the appearance of an XPS peak originating from the -COOgroups (Supplementary Fig. 4). In addition, the amount of the -OH groups at the $\mathrm{SnO}_{2}$ surface was reduced by $\sim 60 \%$ after the CPTA-SAM formation (Supplementary Fig. 4).
To check the influence of deactivating the $-\mathrm{OH}$ groups by the CPTA-SAM on the $J-V$ hysteresis, we fabricated PSCs in the presence and absence of the SAM treatment. PSCs without the CPTA-SAM had the pronounced $J-V$ hysteresis (Supplementary Fig. 5). This was consistent with the previous result shown in Fig. 1b. In contrast, adding the CPTA-SAM significantly reduced the $J-V$ hysteresis of PSCs (Fig. $3 \mathrm{~b}$ and Supplementary Table 3 ). The average PCEs of eight devices with the CPTA-SAM were $18.06 \%$ and $18.12 \%$ in the forward and reverse scans, respectively (Supplementary Table 4). In addition, no hysteresis was observed in PSCs with the CPTA-SAM even at a high temperature of $60{ }^{\circ} \mathrm{C}$ (Fig. 3c). Furthermore, there was no increase in the size of the semicircle in EIS results shown in Fig. 3d.

The improved electron extraction to $\mathrm{SnO}_{2}$ by the use of the CPTA-SAM is likely for the reduced hysteresis on the basis of literature ${ }^{43,44}$. Therefore, we prepared additional PSCs using a thin layer of pure $\mathrm{C}_{60}$. Using this $\mathrm{C}_{60}$ layer can distinguish whether the decreased hysteresis resulted from the improved electron extraction or reduced localization of the positive ions because the surface $-\mathrm{OH}$ groups still exist below a $\mathrm{C}_{60}$ layer. As shown in Supplementary Fig. 6, the PL intensity reduction was similar when a perovskite film was prepared on the $C_{60}$ layer or 
the CPTA-SAM, indicating the similar electron extraction efficiency in both the cases. The reduced PL intensities also pointed to the existence of $\mathrm{C}_{60}$ even after the spin coating of the perovskite film. The pronounced $J-V$ hysteresis was observed when $\mathrm{C}_{60}$ was used in PSCs (Fig. 3e and Supplementary Tables 5 and 6), despite the similar electron extraction. This indicates that the deactivation of the surface $-\mathrm{OH}$ groups is very important for reducing the hysteresis. Furthermore, the CPTA-SAM and the $\mathrm{C}_{60}$ layer could have different dipole moments, which may affect the ion localization and the PSC performance.

In addition to the ion migration, unbalanced charge transport $^{45-47}$, ferroelectricity ${ }^{47,48}$, capacitive effect ${ }^{47,49}$, and defectinduced electronic traps ${ }^{50}$ are known to induce the $J-V$ hysteresis. There is a possibility that all these factors changed in our PSCs simultaneously when the $\mathrm{SnO}_{2}$ surfaces were treated differently, although every perovskite film fabricated on the untreated, CPTA-treated, and $\mathrm{C}_{60}$-coated surfaces had similar morphologies (Supplementary Fig. 7). We will carry out experiments in our laboratory to clarify the more detailed origins of the $J-V$ hysteresis.

Long-term stability under continuous light illumination. We measured the stability of PSCs with and without the CPTA-SAM under continuous illumination with an intensity of $100 \mathrm{~mW} \mathrm{~cm}^{-2}$ near a maximum power point. Figure 4 a shows the PCE evolution of PSCs over time, and other parameters of $J_{\mathrm{SC}}, V_{\mathrm{OC}}$, and fill factor (FF) are shown in Supplementary Fig. 8. The PCEs of PSCs without the CPTA-SAM significantly dropped. It is worth mentioning here that PSCs with the CPTA-SAM had no decrease of the PCE after $1000 \mathrm{~h}$ of the continuous illumination. We also investigated the stability of PSCs with the $\mathrm{C}_{60}$ layer. There was no improvement in stability when using $\mathrm{C}_{60}$ (Supplementary Fig. 9). Furthermore, we obtained the improved stability with the CPTASAM under the illumination even at a high temperature of $60^{\circ} \mathrm{C}$ (Fig. $4 \mathrm{~b}$ and Supplementary Fig. 10). These results clearly demonstrated the importance of using the surface treatment for fabricating stable PSCs.

In summary, we found that the $J-V$ hysteresis and degradation can be suppressed by chemically modifying the $\mathrm{SnO}_{2}$ surface with a SAM. We speculated that one reason for the suppressed hysteresis and degradation is the deactivation of the surface $-\mathrm{OH}$ groups, which attract the positive ions. We obtained almost no degradation in PSCs with CPTA-SAM after $1000 \mathrm{~h}$ of continuous illumination at room temperature. Additionally, the introduction of CPTA-SAM greatly improved the high-temperature stability. These findings will provide insights into mechanisms of the hysteresis and long-term stability of PSCs.

\section{Methods}

Materials. A $\mathrm{SnO}_{2}$ colloidal precursor solution [tin(IV) oxide, $15 \%$ in $\mathrm{H}_{2} \mathrm{O}$ colloidal dispersion] was purchased from Alfa Aesar. CPTA was purchased from Sigma-Aldrich. All precursor materials, such as cesium iodide (CsI), formamidinium iodide (FAI), methylammonium bromide (MABr), lead iodide $\left(\mathrm{PbI}_{2}\right)$, and lead bromide $\left(\mathrm{PbBr}_{2}\right)$, for the perovskite fabrication were purchased from Tokyo Chemical Industry. Spiro-OMeTAD was purchased from Merck. The solvents, such as chlorobenzene, DMF, and dimethyl sulfoxide (DMSO), and the dopants for the hole transport material, such as lithium bis(tri-

fluoromethanesulfonyl)imide (LiTFSI), 4-tert-butylpyridine (4-tBP), and FK-209 (tris(2-(1H-pyrazol-1-yl)-4-tert-butylpyridine)cobalt(III) tri[bis(trifluoromethane) sulfonimide]), were purchased from Sigma-Aldrich. These purchased materials were used as received without further purification.

Device fabrication. Glass substrates coated with a pre-patterned ITO layer with a thickness of $100 \mathrm{~nm}$ (Atsugi Micro, Japan) and a sheet resistance of $10 \Omega \mathrm{sq}^{-1}$ were cleaned sequentially by ultrasonication in detergent, pure water, acetone, and isopropanol for $10 \mathrm{~min}$ each and then subjected to ultraviolet (UV)-ozone treatment for 15 min. Next, a $\mathrm{SnO}_{2}$ film was deposited on the ITO surface by spin coating at 3000 r.p.m. for $30 \mathrm{~s}$ using aqueous $\mathrm{SnO}_{2}$ colloidal solution, which was diluted to $2.67 \%$ in water. This $\mathrm{SnO}_{2}$ film was annealed at $150^{\circ} \mathrm{C}$ for $30 \mathrm{~min}$ to dry and treated with UV-ozone for $15 \mathrm{~min}$. Then, the CPTA-SAM was formed on the $\mathrm{SnO}_{2}$ surface by spin coating at 4000 r.p.m. for $30 \mathrm{~s}$ from CPTA solution in DMF with a concentration of $0.5 \mathrm{mg} \mathrm{m}^{-1}$. To remove physiosorbed CPTA molecules from the $\mathrm{SnO}_{2}$ surface, the substrate was washed by DMF and dried at $100{ }^{\circ} \mathrm{C}$ for $5 \mathrm{~min}$. The CsFAMA perovskite layer was prepared according to the literature 4 . Specifically, precursor solution used here was prepared by mixing FAI $(1.10 \mathrm{M})$, $\mathrm{PbI}_{2}(1.16 \mathrm{M}), \mathrm{MABr}(0.2 \mathrm{M})$, and $\mathrm{PbBr}_{2}(0.2 \mathrm{M})$, each of which was dissolved in anhydrous DMF/DMSO (4:1 vol/vol). Additionally, $0.08 \mathrm{M}$ CsI solution in DMSO was added into the aforementioned precursor solution. The precursor solution was stirred at $70^{\circ} \mathrm{C}$ for $3 \mathrm{~h}$ and then filtered with a $0.2 \mu \mathrm{m}$ polytetrafluoroethylene filter before use. Using this solution, a perovskite film was spin coated on the untreated and treated $\mathrm{SnO}_{2}$ surfaces at 1000 r.p.m. for $10 \mathrm{~s}$ and then at 6000 r.p.m. for $30 \mathrm{~s}$. Ten seconds before the end of the substrate rotation, $120 \mu \mathrm{l}$ of chlorobenzene was dropped onto the spinning substrate. Subsequently, the perovskite layer was annealed at $100^{\circ} \mathrm{C}$ for $45 \mathrm{~min}$. The spiro-OMeTAD layer was deposited on top of the perovskite layer by spin coating at 4000 r.p.m. for $30 \mathrm{~s}$ from solution of spiroOMeTAD (36.5 mg in $0.5 \mathrm{ml}$ chlorobenzene). This solution was doped with $15 \mu \mathrm{l}$ of 4-tBP stock solution ( $520 \mathrm{mg} \mathrm{ml}^{-1}$ in acetonitrile), $9 \mu \mathrm{l}$ of LiTFSI stock solution $\left(520 \mathrm{mg} \mathrm{ml}^{-1}\right.$ in acetonitrile), and $14.5 \mu \mathrm{l}$ of FK-209 stock solution $\left(300 \mathrm{mg} \mathrm{ml}^{-1}\right.$ in acetonitrile). The preparation of perovskite and spiro-OMeTAD was done in a nitrogen-filled glove box, while the $\mathrm{SnO}_{2}$ films were fabricated in air. Finally, a 100nm-thick gold electrode was thermally deposited in vacuum to complete the devices. The deposition rate was $0.1 \mathrm{~nm} \mathrm{~s}^{-1}$ and the base pressure was $10^{-4} \mathrm{~Pa}$ for the Au deposition. The fabricated PSCs were encapsulated using a glass lid and UV-cured sealant.

Characterization. $J-V$ curve measurements were performed on the PSCs using a computer-controlled Keithley 2400 source unit under simulated AM1.5 G solar illumination from a Xe lamp-based solar simulator (SRO-25 GD, Bunko-Keiki, Japan) with a scan rate of $200,100,20$, or $10 \mathrm{mV} \mathrm{s}^{-1}$. EIS measurements were performed using a Solartron 1260 frequency-response analyzer from $1 \mathrm{MHz}$ to $70 \mathrm{mHz}$ at an excitation amplitude of $20 \mathrm{mV}$. While the original PSC area defined by the overlap of the ITO and Au electrodes was $4 \mathrm{~mm}^{2}$, the illumination area during the $J-V$ measurements was defined at $3.24 \mathrm{~mm}^{2}\left(1.8 \times 1.8 \mathrm{~mm}^{2}\right)$ using a black shadow mask. The lamp power was carefully calibrated at $100 \mathrm{~mW} \mathrm{~cm}{ }^{-2}$ (1 Sun) using a crystalline Si reference cell with an amorphous Si optical filter (Bunko-Keiki), which was certificated by the National Institute of Advanced Industrial Science and Technology of Japan.

The HI was calculated by the following formula:

$$
\mathrm{HI}=\frac{\mathrm{PCE}_{\text {reverse }}-\mathrm{PCE}_{\text {forward }}}{\mathrm{PCE}_{\text {reverse }}},
$$

where $\mathrm{PCE}_{\text {reverse }}$ and $\mathrm{PCE}_{\text {forward }}$ are the PCE values measured in the forward and reverse bias scans, respectively. The calculated HI values are summarized in Supplementary Tables 1-10.
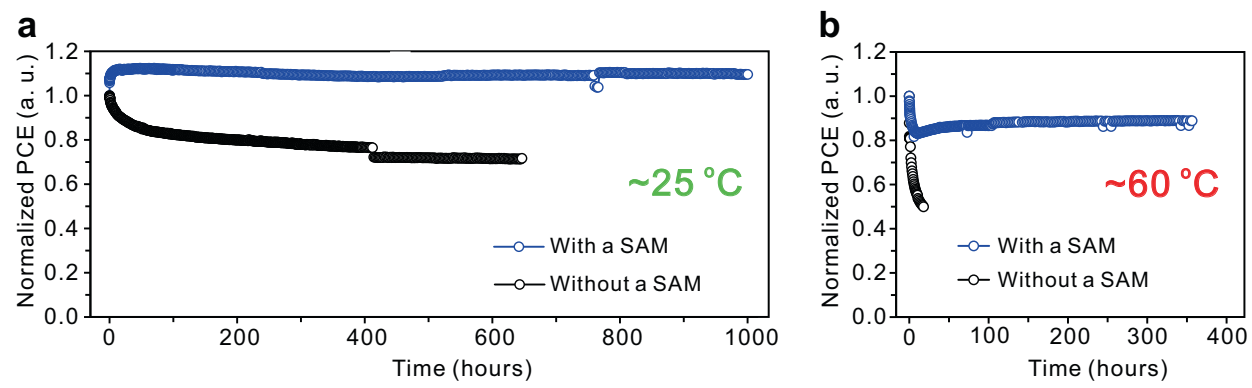

Fig. 4 Addition of a self-assembled monolayer (SAM) improves long-term stability of perovskite solar cells. Efficiency evolution of perovskite solar cells at room temperature $\left(25^{\circ} \mathrm{C}\right)$ (a) and at elevated temperature $\left(60^{\circ} \mathrm{C}\right)(\mathbf{b})$ 
Device lifetime measurements. Light from a white light-emitting diode array with an intensity of $100 \mathrm{~mW} \mathrm{~cm}^{-2}$ was continuously illuminated on the encapsulated PSCs in ambient air at room temperature $\left(25^{\circ} \mathrm{C}\right)$ or $60^{\circ} \mathrm{C}$. The evolution of $V_{\mathrm{OC}}$, $J_{\mathrm{SC}}, \mathrm{FF}$, and PCE was automatically measured using a lifetime measurement system (System Engineers). In between scans, the PSC was connected with a $1 \mathrm{k} \Omega$ resistor ${ }^{22}$.

Steady-state PL measurements. PL spectra of perovskite films were obtained from a JASCO FP-8600 spectrofluorometer in the wavelength range from 600 to $900 \mathrm{~nm}$ with a wavelength step of $0.2 \mathrm{~nm}$. The excitation wavelength used here was $460 \mathrm{~nm}$.

Temperature-dependent conductivity measurements. Temperature-dependent conductivity measurements were carried out using a probe station (Lakeshore, TTP4) equipped with an environmental chamber and thermoelectric plate. The temperature of the device was controlled by either heating with a thermoelectric plate or cooling with a flow of liquid nitrogen. Electrical measurements were conducted using a source/measurement unit (Agilent, B2902A). A lateral device with a configuration of $\mathrm{Au} /$ perovskite $(100 \mu \mathrm{m}) / \mathrm{Au}$ was used for the measurement. The distance between the two Au electrodes was $100 \mu \mathrm{m}$.

Reporting summary. Further information on research design is available in the Nature Research Reporting Summary linked to this article.

\section{Data availability}

The data that support the findings of this study are available from the corresponding author upon reasonable request.

Received: 14 October 2019; Accepted: 16 April 2020;

Published online: 22 May 2020

\section{References}

1. Miyasaka, T., Kojima, A., Teshima, K. \& Shirai, Y. Organometal halide perovskites as visible-light sensitizers for photovoltaic cells. J. Am. Chem. Soc. 131, 6050-6051 (2009).

2. Pellet, N. et al. Mixed-organic-cation perovskite photovoltaics for enhanced solar-light harvesting. Angew. Chem. Int. Ed. 53, 3151-3157 (2014),

3. Jeon, N. J. et al. Compositional engineering of perovskite materials for highperformance solar cells. Nature 517, 476-480 (2015)

4. Saliba, M. et al. Cesium-containing triple cation perovskite solar cells: improved stability, reproducibility and high efficiency. Energy Environ. Sci. 9, 1989-1997 (2016).

5. Jeon, N. J. et al. A fluorene-terminated hole-transporting material for highly efficient and stable perovskite solar cells. Nat. Energy 3, 682-689 (2018).

6. Jiang, Q. et al. Surface passivation of perovskite film for efficient solar cells. Nat. Photonics 13, 500 (2019).

7. National Renewable Energy Laboratory. Best Research-Cell Efficiency Chart. https://www.nrel.gov/pv/assets/pdfs/best-research-cell-efficiencies.20190802. pdf (2019).

8. Eames, C. et al. Ionic transport in hybrid lead iodide perovskite solar cells. Nat. Commun. 6, 2-9 (2015)

9. Tress, W. et al. Understanding the rate-dependent $J-V$ hysteresis, slow time component, and aging in $\mathrm{CH}_{3} \mathrm{NH}_{3} \mathrm{PbI}_{3}$ perovskite solar cells: The role of a compensated electric field. Energy Environ. Sci. 8, 995-1004 (2015).

10. Meloni, S. et al. Ionic polarization-induced current-voltage hysteresis in $\mathrm{CH}_{3} \mathrm{NH}_{3} \mathrm{PbX}_{3}$ perovskite solar cells. Nat. Commun. 7, 10334 (2016).

11. Meng, L., You, J. \& Yang, Y. Addressing the stability issue of perovskite solar cells for commercial applications. Nat. Commun. 9, 5265 (2018).

12. $\mathrm{Bu}, \mathrm{T}$. et al. A novel quadruple-cation absorber for universal hysteresis elimination for high efficiency and stable perovskite solar cells. Energy Environ. Sci. 10, 2509-2515 (2017).

13. Xing, J. et al. Ultrafast ion migration in hybrid perovskite polycrystalline thin films under light and suppression in single crystals. Phys. Chem. Chem. Phys. 18, 30484-30490 (2016).

14. Hou, Y. et al. Suppression of hysteresis effects in organohalide perovskite solar cells. Adv. Mater. Interfaces 4, 1700007 (2017).

15. Miyano, K., Yanagida, M., Tripathi, N. \& Shirai, Y. Hysteresis, stability, and ion migration in lead halide perovskite photovoltaics. J. Phys. Chem. Lett. 7, 2240-2245 (2016).

16. Weber, S. A. L. et al. How the formation of interfacial charge causes hysteresis in perovskite solar cells. Energy Environ. Sci. 11, 2404-2413 (2018)
17. Sherkar, T. S. et al. Recombination in perovskite solar cells: Significance of grain boundaries, interface traps, and defect ions. ACS Energy Lett. 2, 1214-1222 (2017).

18. Domanski, K. et al. Migration of cations induces reversible performance losses over day/night cycling in perovskite solar cells. Energy Environ. Sci. 10, 604-613 (2017).

19. Futscher, M. H. et al. Electronic properties of hybrid organic/inorganic semiconductor pn-junctions. J. Phys. Condens. Matter 31, 064002 (2019).

20. Lee, J. W. et al. 2D perovskite stabilized phase-pure formamidinium perovskite solar cells. Nat. Commun. 9, 1-10 (2018).

21. Zhou, W. et al. Constructing $\mathrm{CsPbBr}_{3}$ cluster passivated-triple cation perovskite for highly efficient and operationally stable solar cells. Adv. Funct. Mater. 29, 1-11 (2019).

22. Yang, S. et al. Stabilizing halide perovskite surfaces for solar cell operation with wide-bandgap lead oxysalts. Science 365, 473-478 (2019).

23. Bai, S. et al. Planar perovskite solar cells with long-term stability using ionic liquid additives. Nature 571, 245-250 (2019).

24. Cao, J., Tao, S. X., Bobbert, P. A., Wong, C. P. \& Zhao, N. Interstitial occupancy by extrinsic alkali cations in perovskites and its impact on ion migration. Adv. Mater. 30, 1-9 (2018).

25. Jung, K. H., Seo, J. Y., Lee, S., Shin, H. \& Park, N. G. Solution-processed $\mathrm{SnO}_{2}$ thin film for a hysteresis-free planar perovskite solar cell with a power conversion efficiency of 19.2\%. J. Mater. Chem. A 5, 24790-24803 (2017).

26. Méndez, P. F., Muhammed, S. K. M., Barea, E. M., Masi, S. \& Mora-Seró, I. Analysis of the UV-ozone-treated $\mathrm{SnO}_{2}$ electron transporting layer in planar perovskite solar cells for high performance and reduced hysteresis. Sol. RRL 3 , 1900191 (2019).

27. $\mathrm{Wu}, \mathrm{F}$. et al. Bias-dependent normal and inverted $J-V$ hysteresis in perovskite solar cells. ACS Appl. Mater. Interfaces 10, 25604-25613 (2018).

28. Tress, W. Metal halide perovskites as mixed electronic-ionic conductors: challenges and opportunities-from hysteresis to memristivity. J. Phys. Chem. Lett. 8, 3106-3114 (2017).

29. Nemnes, G. A. et al. How measurement protocols influence the dynamic $J-V$ characteristics of perovskite solar cells: theory and experiment. Sol. Energy 173, 976-983 (2018).

30. Jiang, Y., Feng, Y., Sun, X., Qin, R. \& Ma, H. Identifying inverted-hysteresis behavior of $\mathrm{CH}_{3} \mathrm{NH}_{3} \mathrm{PbI}_{3-x} \mathrm{Cl}_{x}$ planar hybrid perovskite solar cells based on external bias precondition. J. Phys. D 52, 385501 (2019).

31. Zou, Y. \& Holmes, R. J. Temperature-dependent bias poling and hysteresis in planar organo-metal halide perovskite photovoltaic cells. Adv. Energy Mater. 6, 1501994 (2016)

32. Abdullah, H. Z., Amirnordin, S. H., Ab. Aziz, S. A. B., Rahman, H. A. \& Taib, $\mathrm{H}$. Effect of zeta potential of stanum oxide $\left(\mathrm{SnO}_{2}\right)$ on electrophoretic deposition (EPD) on porous alumina. Adv. Mater. Res. 795, 334-337 (2013).

33. Han, G. S. et al. Spin coating process for $10 \mathrm{~cm} \times 10 \mathrm{~cm}$ perovskite solar modules enabled by self-assembly of $\mathrm{SnO}_{2}$ nanocolloids. ACS Energy Lett. 4, 1845-1851 (2019).

34. Donley, C. et al. Characterization of indium-tin oxide interfaces using X-ray photoelectron spectroscopy and redox processes of a chemisorbed probe molecule: effect of surface pretreatment conditions. Langmuir 18, 450-457 (2002).

35. Jadhav, H., Suryawanshi, S., More, M. A. \& Sinha, S. Pulsed laser deposition of tin oxide thin films for field emission studies. Appl. Surf. Sci. 419, 764-769 (2017).

36. Snaith, H. J. et al. Microseconds, milliseconds and seconds: deconvoluting the dynamic behaviour of planar perovskite solar cells. Phys. Chem. Chem. Phys. 19, 5959-5970 (2017).

37. Klotz, D., Tumen-Ulzii, G., Qin, C., Matsushima, T. \& Adachi, C. Detecting and identifying reversible changes in perovskite solar cells by electrochemical impedance spectroscopy. RSC Adv. 9, 33436-33445 (2019).

38. Calado, P. et al. Evidence for ion migration in hybrid perovskite solar cells with minimal hysteresis. Nat. Commun. 7, 1-10 (2016).

39. Pockett, A. \& Carnie, M. J. Ionic influences on recombination in perovskite solar cells. ACS Energy Lett 2, 1683-1689 (2017).

40. Wang, Y. C. et al. Efficient and hysteresis-free perovskite solar cells based on a solution processable polar fullerene electron transport layer. Adv. Energy Mater 7, 1-10 (2017).

41. Zhong, M. et al. Highly efficient flexible $\mathrm{MAPbI}_{3}$ solar cells with a fullerene derivative-modified $\mathrm{SnO}_{2}$ layer as the electron transport layer. J. Mater. Chem. A 7, 6659-6664 (2019).

42. Chen, J., Zhao, X., Kim, S. \& Park, N. Multifunctional chemical linker imidazoleacetic acid hydrochloride for $21 \%$ efficient and stable planar perovskite solar cells. Adv. Mater. 31, 1902902 (2019).

43. Carrillo, J. et al. Ionic reactivity at contacts and aging of methylammonium lead triiodide perovskite solar cells. Adv. Energy Mater 6, 1-7 (2016). 
44. Shao, Y., Xiao, Z., Bi, C., Yuan, Y. \& Huang, J. Origin and elimination of photocurrent hysteresis by fullerene passivation in $\mathrm{CH}_{3} \mathrm{NH}_{3} \mathrm{PbI}_{3}$ planar heterojunction solar cells. Nat. Commun. 5, 1-7 (2014).

45. Jiang, Q. et al. Enhanced electron extraction using $\mathrm{SnO}_{2}$ for high-efficiency planar-structure $\mathrm{HC}\left(\mathrm{NH}_{2}\right)_{2} \mathrm{PbI}_{3}$-based perovskite solar cells. Nat. Energy 2, 16177 (2017).

46. Yang, D. et al. High efficiency planar-type perovskite solar cells with negligible hysteresis using EDTA-complexed $\mathrm{SnO}_{2}$. Nat. Commun. 9, 3239 (2018).

47. Chen, B., Yang, M., Priya, S. \& Zhu, K. Origin of $J-V$ hysteresis in perovskite solar cells. J. Phys. Chem. Lett. 7, 905-917 (2016).

48. Wei, J. et al. Hysteresis analysis based on the ferroelectric effect in hybrid perovskite solar cells. J. Phys. Chem. Lett. 5, 3937-3945 (2014).

49. Chen, B. et al. Impact of capacitive effect and ion migration on the hysteretic behavior of perovskite solar cells. J. Phys. Chem. Lett. 6, 4693-4700 (2015).

50. Lee, J. W. et al. The interplay between trap density and hysteresis in planar heterojunction perovskite solar cells. Nano Lett. 17, 4270-4276 (2017).

\section{Acknowledgements}

This work was supported by the Japan Science and Technology Agency (JST), ERATO, Adachi Molecular Exciton Engineering Project (JST ERATO Grant number

JPMJER1305), by the International Institute for Carbon-Neutral Energy Research (WPII2CNER) sponsored by the Ministry of Education, Culture, Sports, Science, and Technology (MEXT) of Japan, by JSPS KAKENHI (Grant numbers JP15K14149, JP16H04192, and 20H02817), and by The Canon Foundation.

\section{Author contributions}

G.T.-U., T.M., and C.A. conceived the idea of this study. G.T.-U. designed and performed experiments such as device fabrication and characterization. D.K. and G.T.-U. conducted EIS measurements. P.W., M.R.L., and G.T.-U. conducted morphological study. C.Q.,

J.W.L., S.-J.L., and Y.Y. conducted temperature-dependent conductivity measurements.
All authors discussed the results and commented on the manuscript. T.M. and C.A. supervised the project.

\section{Competing interests}

The authors declare no competing interests.

\section{Additional information}

Supplementary information is available for this paper at https://doi.org/10.1038/s43246 020-0028-z.

Correspondence and requests for materials should be addressed to C.A.

Reprints and permission information is available at http://www.nature.com/reprints

Publisher's note Springer Nature remains neutral with regard to jurisdictional claims in published maps and institutional affiliations.

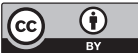

Open Access This article is licensed under a Creative Commons Attribution 4.0 International License, which permits use, sharing, adaptation, distribution and reproduction in any medium or format, as long as you give appropriate credit to the original author(s) and the source, provide a link to the Creative Commons license, and indicate if changes were made. The images or other third party material in this article are included in the article's Creative Commons license, unless indicated otherwise in a credit line to the material. If material is not included in the article's Creative Commons license and your intended use is not permitted by statutory regulation or exceeds the permitted use, you will need to obtain permission directly from the copyright holder. To view a copy of this license, visit http://creativecommons.org/ licenses/by/4.0/.

(C) The Author(s) 2020 